Service social

\title{
Éléments d'approche intégrée en travail social : analyse d'une expérience en milieu scolaire
}

\section{Christine Ouellet et Pierre Richard}

Volume 36, numéro 2-3, 1987

Approches intégrées

URI : https://id.erudit.org/iderudit/706369ar

DOI : https://doi.org/10.7202/706369ar

Aller au sommaire du numéro

Éditeur(s)

École de service social de l'Université Laval

ISSN

1708-1734 (numérique)

Découvrir la revue

Citer cet article

Ouellet, C. \& Richard, P. (1987). Éléments d'approche intégrée en travail social : analyse d'une expérience en milieu scolaire. Service social, 36(2-3), 387-396. https://doi.org/10.7202/706369ar
Résumé de l'article

À partir d'une situation d'intégration scolaire à l'école du village, les auteurs montrent les limites de la pratique du cas par cas et mettent de l'avant le besoin d'un renouveau dans la pratique sociale.

Une expérience de concertation de différents établissements socio-sanitaires et scolaires montre les possibilités de modification des approches et l'élaboration de modes d'interventions institutionnels différents. 
Ouellet, Christine, agente de développement à l'Office des personnes handicapées du Québec, Sherbrooke.

RICHARD, Pierre, coordonnateur au

C.L.S.C. Fleur-de Lys, Weedon.

\section{Éléments d'approche intégrée en travail social : analyse d'une expérience en milieu scolaire}

\section{Christine Ouellet Pierre Richard}

Le but du présent article est d'indiquer, modestement, de quelle façon la pratique sociale est en train d'évoluer au Québec. À partir d'une expérience mettant à contribution différents organismes sociosanitaires et scolaires, nous voulons démontrer que le travail social peut et doit modifier ses approches et modes d'intervention institutionnels en essayant d'allier services individuels, services à des groupes et services d'organisation communautaire.

\section{Un projet d'intégration scolaire à l'école du village}

Le projet d'intégration scolaire sur le territoire de la municipalité de Weedon, origine des besoins d'un enfant handicapé, d'âge scolaire, qui ne recevait aucun service du Ministère de l'Éducation du Québec (M.E.Q.), et bénéficiait seulement de quelques heures d'assistance éducative à domicile offerte par le Centre de réadaptation de la région.

Le projet débute en septembre 1985. À cette époque, dans notre région comme dans plusieurs autres, on se demande encore si les commissions scolaires sont tenues d'offrir des services à tous les enfants, malgré l'obligation en ce sens incluse dans la Loi de l'instruction publique, et de favoriser le maintien dans le milieu de vie naturel. Dans 
notre région, on préférait que l'enfant fréquente l'école spécialisée quand il n'y avait pas nécessité de l'inscrire à la classe spéciale du centre d'accueil ou dans une école au niveau supra-régional. Il arrivait même que, refusé à l'école, l'enfant se retrouve à la charge totale de ses parents, recevant, à domicile, des services de réadaptation du Ministère de la Santé et des Services Sociaux (M.S.S.S.), mais toujours en quantité insuffisante. Pour les enfants handicapés, l'intégration scolaire est malheureusement très difficile; la mentalité des intervenants sociaux et scolaires et de plusieurs membres de la collectivité reste à modifier. Le parent qui désire des services réguliers pour son enfant, doit alors frapper à plusieurs portes et s'armer de patience et de courage.

C'est précisément ce qu'ont vécu les parents de Francis qui, en septembre 1984, a sept ans. Le diagnostic au sujet de l'enfant n'est pas clair; on parle alors d'un retard global de développement. Francis est inscrit à l'école spécialisée régionale par le Centre de réadaptation et il est refusé. Une tentative de scolarisation est faite à la classe spéciale d'un centre d'accueil de Sherbrooke. Cette solution nécessite l'hébergement de l'enfant en famille d'accueil. Le placement ne dure que deux semaines puisque la famille d'accueil ne peut poursuivre. De plus, la famille naturelle constate que ce placement perturbe l'enfant. La mère demande donc à l'Office des personnes handicapées du Québec (O.P.H.Q.) d'intervenir dans la recherche de services pouvant combler les besoins de l'enfant.

L'O.P.H.Q. et le centre local de services communautaires (C.L.S.C.) de cette région, sensibles aux besoins de l'enfant et de la famille, convoquent tous ceux qui connaissent la situation et/ou qui pourraient y jouer un rôle actif. Participent à cette rencontre: le directeur de l'école du village, le représentant de la Commission Scolaire, les travailleuses sociales du Centre Hospitalier, du C.L.S.C. et du Centre de réadaptation. Cette rencontre porte principalement sur l'histoire de Francis. Tous s'entendent alors sur une version commune des besoins de l'enfant et de sa famille et sur l'urgence d'agir.

Avant d'élaborer un plan d'action, les intervenants, les parents et l'école se demandent si la présente démarche ne pourrait pas convenir et servir à d'autres enfants handicapés du milieu et éprouvant les mêmes difficultés. On dénombre trois autres enfants admissibles au projet. Ceux-ci reçoivent de l'assistance éducative à domicile et doivent être orientés vers la maternelle en septembre 1986.

Les participants s'entendent sur les diverses modalités du projet. Les enfants recevront les services éducatifs à l'école. Francis bénéficiera d'un accompagnateur rémunéré par l'O.P.H.Q. Le travailleur social du C.L.S.C. assurera la coordination des plans de services des enfants. Le 
Centre de réadaptation donnera les services d'assistance éducative dans le local disponible de l'école et mettra à la disposition des enfants certains équipements permettant de répondre adéquatement à leurs besoins. Un suivi sera assuré par l'Association de la Paralysie Cérébrale et par le Centre Hospitalier Universitaire de Sherbrooke (C.H.U.S.). De plus, les parents solliciteront l'aide du Club Lions et de la Caisse populaire du village. On impliquera donc la collectivité.

Les enfants arrivent à l'école en octobre 1985. Afin d'assurer le suivi et l'évolution du projet, des rencontres régulières ont lieu entre les intervenants et les parents. Ces derniers veulent en connaître davantage sur leurs droits et désirent discuter de leur situation. Une série de dix rencontres est élaborée et dispensée aux parents par une animatrice, dans le cadre d'une formation sur mesure, donnée par le service de l'Éducation des adultes.

Déjà en février 1986, le comité évalue l'expérience et planifie la prochaine année. Il faut trouver les fonds qui permettraient de rendre l'école accessible. Une demande est acheminée au M.E.Q. De plus, on implique la garderie de Weedon pendant les vacances et on demande au C.H.U.S. de fournir un diagnostic complet sur Francis.

La période d'été débute. Les enfants ont reçu des services à l'école durant l'année et il est important qu'ils puissent s'intégrer aux activités estivales offertes aux autres enfants du village. Les intervenants reconnaissent ce besoin et s'impliquent à nouveau. Les enfants participent aux activités de l'O.T.J. (organisation des terrains de jeux) en bénéficiant du service d'un accompagnateur octroyé par l'O.P.H.Q. Ils continuent de recevoir les services du Centre de réadaptation et fréquentent la garderie. Les familles sont satisfaites dans la mesure où les jeunes conservent leurs acquis tout en continuant l'intégration dans leur milieu. D'autre part, elles ne se sentent plus seules dans leur situation.

En septembre 1986, la réponse du M.E.Q. est favorable à une subvention permettant à ces enfants l'accessibilité à l'école. Le coût inhérent à l'engagement des accompagnateurs est maintenant partagé entre la Commission Scolaire et l'O.P.H.Q. Le Centre de réadaptation reste présent dans le dossier, tant auprès des familles et des enfants qu'auprès des professeurs et de la garderie. Il assume de plus l'encadrement des accompagnateurs.

Actuellement, le projet se poursuit pour la troisième année. Les personnes impliquées sont confiantes quant à la poursuite de l'objectif ultime: I'intégration sociale de ces enfants dans leur milieu de vie naturel. Les enfants se sont développés et ont un fonctionnement adéquat à l'école; trois d'entre eux fréquentent la classe régulière tandis que le quatrième bénéficie d'un programme d'enseignement 
adapté. La communauté continue à suivre le projet et l'école de Weedon est fière de son implication auprès de cette nouvelle clientèle.

L'expérience de l'intégration scolaire de ce petit groupe d'enfants handicapés a posé des questions fondamentales aux intervenants sociaux de même qu'au milieu scolaire. L'école se sentait un peu à l'étroit dans les limites de la Loi de l'instruction publique : elle a accepté de plein gré de se laisser porter par le projet mis de l'avant par les intervenants du M.S.S.S. Les intervenants sociaux des établissements impliqués ont vite pris conscience, grâce à ce projet, des limites de la pratique sociale actuelle et de la nécessité de trouver de nouvelles voies pour intervenir.

\section{Les limites de la pratique du cas par cas}

Certains auteurs ont déjà traité explicitement des limites d'une approche qui se confinerait à la seule consultation individuelle pour aborder ce genre de problématique. Dans ce qui suit, nous rejoignons plus particulièrement la pensée de Pierre Tremblay et de Marcel Lévesque (1.2), en ce qui concerne les exigences actuelles pour la pratique sociale et la nécessité de trouver de nouvelles voies d'intervention. La pratique traditionnelle demande aux intervenants sociaux de procéder à une analyse individuelle des cas, de faire le diagnostic, d'élaborer un plan d'action et d'intervenir. Pris un à un, tous les cas s'intègrent au système en place et le problème "se règle». Mais, bien que nécessaire et appropriée dans plusieurs situations, cette méthode ne fonctionne pas toujours. En effet, lorsque les ressources offertes à la famille ne sont pas adéquates, lorsque l'école ultra-spécialisée ne peut recevoir l'enfant, lorsque la famille demande à ce que l'enfant demeure dans son milieu, bref quand tout a été essayé, force nous est de croire que ce modèle de pratique a des limites : il doit alors être complété par des interventions de groupe et communautaires. Ainsi, dans la situation qui nous concerne, les cas étudiés étaient tous trop lourds pour chaque établissement concerné : il nous fallait mettre en commun des ressources institutionnelles et professionnelles afin d'offrir des services à chacun des enfants tout en leur permettant de continuer à vivre dans leur village et leur famille.

Différents facteurs nous ont fait prendre conscience des limites de l'approche individuelle. D'abord, ce fut l'insistance des parents à exiger pour eux et leurs enfants des services adaptés "sur place". Ensuite, la limite financière imposée à chaque cas pris isolément : desservir chaque cas individuellement nous apparaissait hors de prix ! Comment aurait-il été possible de négocier deux heures de physiothérapie, une heure 
d'ergothérapie, trois heures d'orthopédagogie et cinq heures d'enseignement auprès de quatre enfants demeurant chacun chez eux ? II nous fallait regrouper les besoins et collectiviser les services. Un dernier facteur nous a incité à repousser l'approche du cas par cas: ce fut l'importance accordée par les intervenants du M.S.S.S. à la notion de maintien dans le milieu de vie naturel. Cette notion, investie des éléments de l'approche communautaire, commande aux travailleurs sociaux de pousser beaucoup plus loin leur approche individuelle afin de maximiser cette notion et de lui permettre de trouver sa réalisation dans les réseaux des personnes qui demandent de l'aide.

\section{Les exigences nouvelles pour la pratique sociale}

Dans ce contexte de rareté des ressources, impliquant le maintien dans le milieu de vie naturel et la collectivisation des services, la pratique sociale est placée devant de nombreux défis, dont le premier est de reconnaître les limites de la pratique individuelle. De plus, il nous faut assumer les limites de nos interventions morcelées, dans chacun de nos établissements. Les travailleurs sociaux qui interviennent auprès d'une même clientèle à partir d'établissements différents ne reconnaissent parfois même pas leur fonction spécifique, tellement leurs préoccupations institutionnelles propres ont teinté la vision des besoins de leurs clientèles communes. Il faut donc développer une vision unifiée des besoins de l'usager.

Le deuxième défi, corollaire du premier, est de voir l'usager non plus comme individu isolé mais comme appartenant à un système qui lui est propre: sa famille, son village, les ressources de son milieu. II s'agit alors de concevoir le rôle actif de chacune des ressources dans la mise en place d'un plan de service pour chaque enfant.

Un troisième défi, et non le moindre, interpelle la pratique professionnelle sur ses bases les plus profondes: la conception de l'expert confidentiel. Il nous faut accepter d'être partenaire et associé à d'autres organismes ou réseaux, car le contexte commande une mise en commun, non seulement des besoins et services des clients, mais aussi des expertises des divers professionnels. En ce qui a trait à notre projet, cet exercice fut difficile, et seul l'objectif de répondre au client, dans un cadre qui rencontre ses exigences, nous a permis d'avancer prudemment sur ce terrain.

Un dernier défi consolide les trois premiers et donne une nouvelle orientation à la pratique sociale : il s'agit de pouvoir mener nos interventions dans une perspective à trois dimensions : l'individu, son groupe 
et la communauté dans laquelle il vit. En ce qui nous concerne, chaque intervenant a dû considérer le client dans ses systèmes propres, mais il avait aussi à se voir lui-même comme partie d'un ou de plusieurs systèmes du client et de la communauté dans laquelle ce projet a évolué.

La nécessité de trouver des voies nouvelles pour intervenir

Dans le cas précis de notre projet, certaines nouvelles pistes nous ont été imposées de l'extérieur, l'évidence de la réalité dépassant parfois l'expertise professionnelle dans chacune de nos institutions respectives. Bien sûr, il nous faut ajuster nos interventions aux structures d'accueil et de distribution de services qui sont en constante évolution ; de plus, les valeurs et les choix de clientèles changent et exigent la mise en place de nouveaux services. Mais à notre avis, il y a plus que cela !

Il est nécessaire de trouver de nouvelles voies pour intervenir parce que les résultats de ces nouvelles tentatives sont intéressants et prometteurs. Dans le cas qui nous concerne, nous savons que tout enfant handicapé a avantage à être mis en contact avec des enfants " ordinaires " car il y gagne en stimulation et en intérêt, accélérant d'autant l'acquisition de nouvelles habiletés et de compétences individuelles. De plus, ce contexte interactionnel permet aux pairs de connaître la réalité du handicap, de considérer comme naturelle la présence d'un fauteuil roulant à l'école, à la garderie, aux terrains de jeux, et de s'impliquer comme partenaires de vie de l'enfant handicapé. Ce rapprochement fournit une occasion d'apprentissage qui aura, à court terme, des effets positifs sur les mentalités et les comportements des individus dans leurs rapports avec la personne handicapée. C'est donc dans l'application même du concept d'intégration sociale qu'une nouvelle façon d'intervenir a pris forme chez les travailleurs sociaux concernés.

\section{Analyse de l'expérience dans une perspective d'approche intégrée en travail social}

Lorsque d'une part, les travailleurs sociaux choisissent d'interroger leur pratique, de centrer leur action sur l'individu, son milieu et sa communauté, lorsque d'autre part, les organismes publics choisissent de collaborer entre eux pour la mise en place de solutions adaptées aux besoins de quelques individus, ces efforts conduisent à la mise en 
application de solutions communautaires qui ont une réelle portée de changement social.

Ce projet dans lequel plusieurs organismes et intervenants se sont impliqués a permis de dégager des aspects multiples et diversifiés de l'intervention sociale. Aussi, il nous apparaît que les principales caractéristiques qui s'en dégagent et que nous analysons ci-après, sont apparentées à celles de l' "approche intégrée" en travail social. II s'agit d'abord d'une " approche communautaire et de plan de service " (comme nous l'avons nommée), qui s'appuie sur une entente inter-établissements, qui adopte des stratégies en fonction de cibles diversifiées, et toujours dans une optique de concertation en vue de la dispensation d'un service adéquat.

\section{Approche communautaire et plan de service}

Le projet d'intégration de quatre enfants handicapés, dans les structures "normales" de l'école, était la réponse aux besoins des individus, en matière d'éducation, de réadaptation, de garderie et de loisirs. II ne s'agissait pas simplement de transférer les enfants du M.S.S.S. au M.E.Q. et de fermer les dossiers. C'est sur la base des besoins des clients, établis à l'intérieur d'un plan de services, que le travail pouvait débuter, et non pas à partir de discours de principes ou de cadre légal du type: "Â quel âge un enfant doit-il passer sous la responsabilité de tel ministère à tel autre?"

Bien sûr, ce qui a attiré l'attention des intervenants de plusieurs établissements, c'est la difficulté d'accès au système scolaire. Mais, estce là l'unique problème? En procédant par une approche plus globale, l'analyse des besoins des enfants nous a révélé que la solution relevait de plusieurs systèmes, respectivement responsables d'un plan spécifique d'intervention à l'intérieur d'un plan de service global.

Pourquoi cette approche est-elle communautaire? Partant des besoins individuels, les solutions auraient pu demeurer individuelles. Cependant, les parents ont décidé de rompre le silence quant à leurs difficultés. Ils ont échangé et ont pris conscience que certaines des solutions individuelles pouvaient être applicables à un projet collectif. De parents peu sûrs et peut-être même honteux de leur situation, ils sont devenus participants, impliqués et fiers. L'école étant devenue une collaboratrice importante, ils pouvaient s'allier d'autres ressources de la communauté. C'est ainsi qu'ils ont demandé l'implication de la garderie, le support financier de certains groupes communautaires et qu'ils ont souhaité publiciser le projet dans le journal du village. Le projet s'est 
alors solidement enraciné dans la communauté au point que les institutions et les établissements pourraient difficilement maintenant se soustraire à leurs engagements.

\section{La concertation inter-établissements}

Chaque établissement concerné par cette problématique avait déjà tenté, à sa façon, des interventions auprès de ces enfants, mais aucun service ne pouvait à lui seul, offrir une solution adéquate à cette situation complexe. Ce contexte a favorisé une meilleure écoute et une disponibilité à l'échange.

Devant les besoins exprimés, les intervenants ont accepté de s'entendre sur quelques principes de base: le libre choix de la personne handicapée ou de son représentant ; le droit des enfants de demeurer dans leur milieu naturel ; le droit de recevoir des services éducatifs dans le cadre le plus normal possible ; la reconnaissance, par les établissements, de leurs responsabilités dans le projet.

Forts de ces principes, les intervenants ont procédé à l'analyse des besoins et à la mise en œuvre des plans d'action. Les difficultés rencontrées ont été résolues grâce à la complicité entre les collaborateurs.

À partir du moment où les établissements concernés (école, C.L.S.C., C.S.S., centre de réadaptation, O.P.H.Q.) ne sentent pas qu'ils se réunissent pour trouver un coupable sur qui déverser toute la responsabilité de la situation, les intervenants peuvent se parler; ensemble et avec les clients, ils ajustent leurs actions dans un continuum de services adéquats pour répondre aux besoins des enfants et de leur famille. Les solutions qui en émergent sont des plus créatrices.

\section{Des éléments stratégiques pertinents}

Devant la rareté des ressources socio-sanitaires actuelles et un certain constat d'échec quant à l'utilisation des ressources lourdes d'hébergement et de placement des enfants, la pratique sociale contemporaine tend à modifier son tir ; elle vise maintenant une collaboration avec des ressources plus légères et un consensus des plans d'intervention auprès des usagers. C'est dans cet esprit que nous pourrions faire une appréciation globale de la formule d'intervention découverte et pratiquée par les intervenants dans ce projet d'intégration sociale.

Ce modèle d'intervention s'est appuyé sur une série de stratégies adaptées au contexte. La première a été de permettre à l'usager de prendre du pouvoir sur sa réalité; l'intervenant l'accompagne alors 
dans cette démarche. Prendre du pouvoir signifie prendre la parole, être écouté, rencontrer des personnes ayant un problème semblable, trouver des appuis thérapeutiques et politiques capables de faire avancer le dossier. Dans le cas qui nous concerne, les intervenants sociaux savaient que des parents mobilisés feraient évoluer le dossier plus vite que toutes les structures institutionnelles en place.

La deuxième stratégie a consisté à faire vivre aux représentants de l'école, l'institution la plus concernée par la mise en place du nouveau service, une expérience de succès ; l'idée a été de permettre à l'école de s'ouvrir sur le milieu en réalisant l'intégration scolaire de quatre enfants handicapés. II importait pour l'école de dire sa volonté d'être une ressource significative dans le milieu. Il fut alors convenu de mettre en place un plan d'action à deux volets. Le premier visait à sensibiliser élèves, professeurs et comité d'école. Le second volet consistait à mettre de l'avant un programme d'information au sujet des enfants handicapés pouvant éventuellement fréquenter l'école de leur village. Ces buts ont été atteints par l'utilisation régulière du journal communautaire local.

La troisième stratégie comportait l'implication maximale des intervenants de tous les organismes concernés. Dès le départ, le projet devint prioritaire pour tous, et chacun trouva beaucoup de renforcement dans un suivi régulier et soutenu du dossier: service rapide et direct auprès des usagers, participation clairement définie pour chacun, suivi régulier avec les parents, coordination du projet par le C.L.S.C.

\section{Conclusion}

Nous pouvons affirmer que le consensus et la collaboration entre les acteurs en présence expliquent la réussite de cette expérience. De plus, la clarté du principe de base (l'enfant demeure dans son milieu naturel et recevra des services à l'école du village) a grandement contribué à orienter les actions des divers intervenants. Chacun y travaillant très fort, le projet a eu des impacts éclatants dans le milieu. Il faut également ajouter que le client est resté propriétaire de sa démarche et qu'on lui reconnaissait la compétence et la capacité de collaborer avec les intervenants. L'objectif ultime était que des services s'organisent par la concertation des établissements et la responsabilisation des clients et des collectivités. 


\section{Références}

1 P. TREMBLAY, Domination technocratique et pratique alternative dans les services sociaux, essai présenté pour l'obtention d'une maîtrise en service social, Université Laval, 1985.

2 M. LÉVESQUE, "La consultation individuelle et ses liens avecl'approche communautaire", Intervention, n० 71, 1985: 43-45.

\section{Bibliographie}

LAFLAMME, M. et coll., Le projet coopératif québécois : un projet social, Chicoutimi, Gaëtan Morin 1982.

LAFOREST, M. et B. REDJEB, Le service social dans les C.S.S. au Québec : une double réalité, Québec, École de Service Social, Université Laval, 1983.

LANGelier, G., "Personnels, directions d'école et parents : des partenaires pour l'intégration ", Apprentissage et socialisation, vol. 9, n 2, $1986: 68-73$.

GoupIL, G. et G. BoutıN, L'intégration scolaire des enfants en difficulté, Montréal, Nouvelle Optique, 1983.

Office des personnes handicapées, À part... égale - L'intégration sociale des personnes handicapées: un défi pour tous, Québec, Direction générale des publications gouvernementales du Ministère des Communications, 1984. 